\title{
What Quality and Safety of Care for Patients Admitted to Clinically Inappropriate Wards: a Systematic Review
}

\author{
Micaela La Regina, $M D^{7}$, Francesca Guarneri, $M D^{2}$, Elisa Romano, $M D^{7}$,

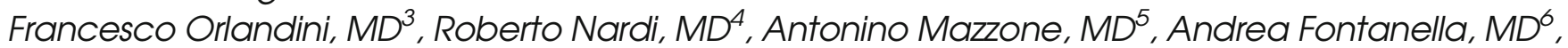 \\ Mauro Campanini, MD', Dario Manfellotto, $\mathrm{MD}^{8}$, Tommaso Bellandi, EurErg PhD', \\ Gualberto Gussoni, $\mathrm{MD}^{10}$, Riccardo Tartaglia, $\mathrm{MD}^{9}$, and Alessandro Squizzato, $\mathrm{MD}^{11}$
}

\begin{abstract}
'SS Risk Management, ASL5 Liguria, La Spezia, Italy; 'Laboratorio Management e Sanita', Institute of Management of Scuola Superiore Sant'Anna of Pisa, Pisa, Italy; ${ }^{3}$ Direzione Sanitaria, ASL4 Liguria, Chiavari, Italy; ${ }^{4}$ Dipartimento Medicina Interna, Bologna, Italy; ${ }^{5}$ Dipartimento Medico Ospedale di Legnano, Azienda Socio Sanitaria Territoriale Ovest Milanese, Legnano, MI, Italy; ${ }^{6}$ Dipartimento di Medicina Interna, Ospedale del Buonconsiglio - Fatebenefratelli Napoli, Naples, NA, Italy; ${ }^{7}$ Dipartimento di Medicina Interna, Azienda Ospedaliera Maggiore della Carità, Novara, Italy; ${ }^{8}$ UO Medicina Interna, Ospedale Fatebenefratelli Isola Tiberina, Rome, Italy; ${ }^{9}$ Centro Gestione Rischio Clinico Regione Toscana, Florence, Italy; ${ }^{10} \mathrm{Centro}$ Studi FADOI, Rome, Italy; "'Dipartimento di Medicina Clinica e Sperimentale, Università dell'Insubria, Varese, Italy.
\end{abstract}

BACKGROUND: In countries with public health system, hospital bed reductions and increasing social and medical frailty have led to the phenomenon of "outliers" or "outlying hospital in-patients." They are often medical patients who, because of unavailability of beds in their clinically appropriate ward, are admitted wherever unoccupied beds are. The present work is aimed to systematically review literature about quality and safety of care for patients admitted to clinically inappropriate wards.

METHODS: We performed a systematic review of studies investigating outliers, published in peer-reviewed journals with no time restrictions. Search and screening were conducted by two independent researchers (MLR and ER). Studies were considered potentially eligible for this systematic review if aimed to assess the quality and/ or the safety of care for patients admitted to clinically inappropriate units. Our search was supplemented by a hand search of references of included studies. Given the heterogeneity of studies, results were analyzed thematically. We used PRISMA guidelines to report our findings.

RESULTS: We collected 17 eligible papers and grouped them into six thematic categories. Despite their methodological limits, the included studies show increased trends in mortality and readmissions among outliers. Quality of care and patient safety are compromised as patients and health professionals declare and risk analysis displays. Reported solutions are often multicomponent, stress early discharge but have not been investigated in the control group.

CONCLUSIONS: Published literature cannot definitely conclude on the quality and safety of care for patients admitted to clinically inappropriate wards. As they may represent a serious threat for quality and safety, and moreover often neglected and under valued, well-designed and powered prospective studies are urgently needed.

KEY WORDS: medical outliers; clinically inappropriate wards; quality assessment; risk assessment; hospital administration.

Received October 10, 2018

Revised November 5, 2018

Accepted December 27, 2018

Published online April 22, 2019
J Gen Intern Med 34(7):1314-21

DOI: $10.1007 / \mathrm{s} 11606-019-05008-4$

(C) Society of General Internal Medicine 2019

\section{INTRODUCTION}

In the last few years, progressive reductions in hospital beds, growing social and medical frailty that impedes hospital discharge, and an inadequate availability of community healthcare services have led to a severe lack of hospital beds. Consequently, emergency physicians are forced to admit patients to clinically inappropriate wards.

The so-called outlier, out-lying hospital in-patient, overflow, sleep-out, or boarder ${ }^{1-3}$ is a patient who, because of unavailability of hospital beds in his/her clinically appropriate ward, is admitted wherever an unoccupied bed is. In such a case, clinical management is provided by the medical staff of the clinically appropriate ward (generally, internal medicine), but care is delivered by nursing staff of the hosting ward. An example is a patient with pneumonia who, because for unavailability of beds in internal medicine, is admitted to a surgical ward.

About $7-8 \%$ of all admissions every year are outlier patients. ${ }^{2}$ The phenomenon is common, particularly in countries with a public health system, and could pose a serious threat for quality and safety of patient care.

The aim of the present work is to systematically review literature evidences about such a phenomenon that is another face of hospital overcrowding.

\section{METHODS}

We performed a systematic review of studies investigating outliers, published in peer-reviewed journals with no time and language restrictions.

We searched Medline/PubMed and EMBASE using the following terms: (("Outlier" OR "out-lying hospital in- 
patient" OR "overflow" OR "sleep-out" OR "boarder" OR "bed-spaced patient" OR "clinically inappropriate ward" AND "mortality", "length of stay", "satisfaction", "adverse event", "medical error", "patient safety")).

The search and screening were conducted by two independent researchers (MLR and ER). Studies were considered potentially eligible for this systematic review if aimed to assess the quality and/or the safety of care for patients admitted to clinically inappropriate units. Our search was supplemented by a hand search of references of included studies. Among them, we found some bed management policies available on hospital websites. They provide recommendations for a safe management of outliers. The search on Medline/PubMed and EMBASE using terms (("bed management" AND "policy" OR "healthcare policy" OR "hospital utilization")) did not produce useful results, so we decided not to include them in our review. Figure 1 shows the process and the results.

Initially, we considered pooling some outcomes (mortality, length of stay, and readmission rates) but abstracted data yielded alarmingly high degrees of heterogeneity $\left(I^{2}>95 \%\right)$, so we decided to analyze our results thematically. Study characteristics were examined to explain differences in findings (Table 1). We used PRISMA guidelines to report our findings.

\section{RESULTS}

Our research retrieved 17 eligible papers, mainly studies conducted on medical patients. We divided them in six thematic categories according to the investigated outcome (details in Table 2a-g).

\section{a. Mortality}

The impact of outlier status on in-hospital mortality was reported in eight studies. Perimal-Lewis et al. ${ }^{9}$ found that being an outlier patient increases the risk-adjusted risk of in-hospital mortality by over $40 \%$ (50\% of deaths happened in the first $48 \mathrm{~h}$ after admission). Bai et al. ${ }^{4}$ reported similar findings: the risk of in-hospital mortality was three times higher among "bed-spaced patients" in the first week just when patients need more interventions. They also suggested several possible reasons for this: less patient contact with physicians on the clinically appropriate ward; inadequate communication between physicians and host-allied health team members; different skills and experience of the allied health team on host ward. Santamaria et al. ${ }^{8}$ reported a mortality increase among outliers in general and Perimal-Lewis et al. ${ }^{6}$ among outliers affected by dementia. These data were refuted by Stowell et al. ${ }^{10}$ and by Stylianou et al. ${ }^{1}$ on large numbers (over 70,000 admissions in 3 years of observation) and by Alameda et al. ${ }^{13}$ among outliers with heart failure. Stowell et al. ${ }^{10}$ and Stylianou et al. ${ }^{1}$ examined also 30-day mortality without finding any increase; Perimal et al..$^{6,9}$ instead revealed a nonsignificant increase in outliers.
Serafini et al. ${ }^{7}$ investigated 3828 consecutive patients hospitalized in medicine and geriatrics in 2012 and, after adjustment for age and sex, the risk of death was about twice as high for outlier patients admitted to surgical area versus the medical one (hazard ratio 1.8, 95\% CI 1.2-2.5).

b. Length of stay (LOS)

LOS was explored in seven studies. Stowell et al. ${ }^{10}$ and Stylianou et al. ${ }^{1}$ found a longer LOS among outlier patients ( 8 vs 7 days and 7 vs 3 days, respectively), consistent with findings by Alameda et al. ${ }^{13}$ among outliers affected by heart failure (11.8 vs 9.2 days). PerimalLewis et al. ${ }^{9}$ registered a significantly shorter length of stay among outliers (110.7 h vs $141.9 \mathrm{~h}$ ). No difference was found by Serafini et al., ${ }^{7}$ either for medicine or geriatrics (10 vs 9.8 days and 13 days for both, respectively) or by Bai et al. (5.31 vs 5.97 days; $p=0.1119){ }^{4}$

c. Readmissions

Readmissions have been studied by five studies; , 6, 7, 9, 10,

${ }^{13}$ Perimal $^{9}$ reported that readmission rates within 7 or 28 days were substantially lower in the outlier group (2.1 vs $1.2 \%$ and $2.1 \%$ vs $4.9 \%$ ). Alameda ${ }^{13}$ found an insignificant increase in readmissions with the same DRG at 30 days among outliers affected by heart failure (15\% vs $10 \%$ ). While a univariate analysis suggested increased hospital admissions, adjustment for various patient characteristics found that outlier status did not affect readmission. ${ }^{1}$ On the other hand, two studies found increased readmission rates; ${ }^{7,10}$ the latter found this to be true in both geriatric $(29.9 \%$ vs $7.2 \%, p<0.0001)$ and general medicine patients $(23.7 \%$ vs $16.3 \%, p=0.01)$.

d. Other indicators

Additional investigated variables include rates of VTE prophylaxis and test ordering, finding that outliers had lower rates of VTE prophylaxis, ${ }^{10}$ though no difference in blood or imaging tests.

ED stay was longer in patients eventually admitted to outlying wards; ${ }^{6}$ respiratory patients were less likely to be outliers than other diseases. ${ }^{7}$

One study ${ }^{11}$ found that the "time burden" from visiting patients on outlying wards was significant, nearly doubling the total time spent with patients, though most of this was due to travel time. In addition to taking more time, another study found that elective operations were reduced by almost $15 \%$ in presence of outliers boarding on the surgical wards. Another study ${ }^{13}$ measured a composite outcome, called "in-hospital morbidity" (intra-hospital infection (urinary, respiratory, bacteremia, or others beginning $48 \mathrm{~h}$ after admission), intra-hospital hemorrhages (digestive, urinary, or others), and intra-hospital venous thromboembolism). Anyway, in-hospital morbidity was found not statistically different between outliers and inliers $(24 \%$ vs $18 \%, p=0.254)$.

On the other hand, outliers were more likely to miss medications ${ }^{12}$ and resulted in increased rates of calls for in-hospital emergency teams. ${ }^{8}$ 


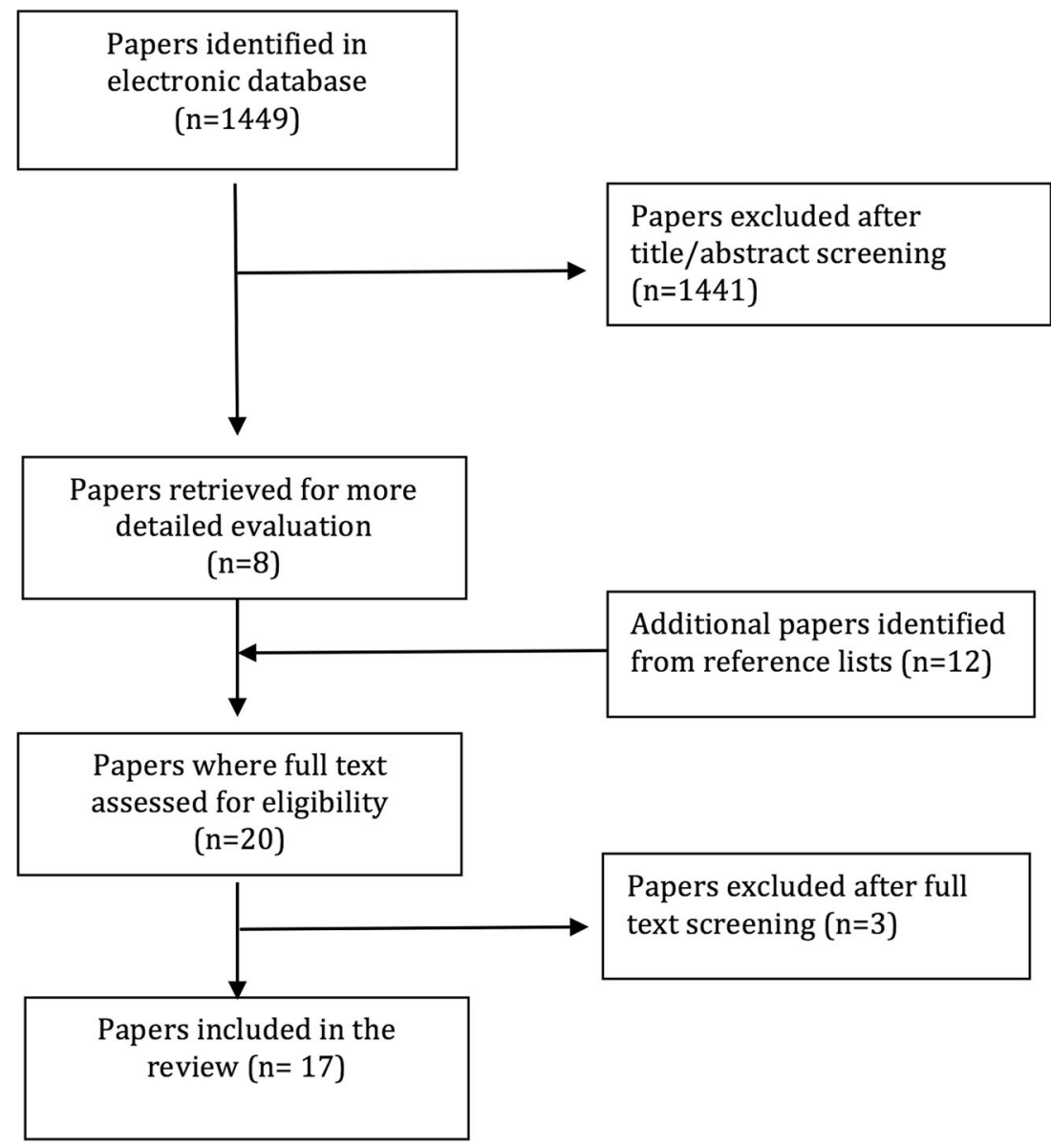

Figure 1 Algorithm of study identification and selection.

e. Perceived quality and safety of care

Goulding et al. explored quality and safety issues from two - the provider and patient- perspectives, ${ }^{2,3}$ finding that both groups were worried. Healthcare providers were concerned about five threats to patient safety: (1) increased workload; (2) poor communication between the two wards; (3) less experience about these patients on clinically inappropriate wards; (4) unsuitable ward environment; (5) characteristics of outlying patients. In addition, patients on inappropriate wards may be perceived as less important and moving patients between wards could disorient older and cognitively impaired patients. ${ }^{3}$ Patients were worried about not belonging, possible communication deficiencies, medical staff availability, nurses' experience, and resource availability. ${ }^{2}$

f. Safety issues and solutions

Four studies evaluated the impact of organizational changes on outliers' risks. One study ${ }^{15}$ suggested solutions such as active discharge planning from the admission, increase of transfers from general internal medicine to geriatrics in another building, and implementation of a consultant-led ward round 7 days a week. Another study instituted a "physician of the week"16 to review outlying patients and improve continuity of care, and added a discharge facilitator and a short stay ward for patients and acutely unstable patients who required a high level of medical care. The study by Lepage et al. ${ }^{14}$ identified five domains of potential failure in the management of outliers: care in emergency department, transfer to the outlying wards, first day of hospital care, care from second day to discharge, day of discharge. They then implemented the following solutions: a doctor, in the clinically appropriate wards, who is in charge of outlying patients each day, a nurse coordinator who facilitates communication between the emergency department, specialty wards, and outlying wards and ensure that the location of outlying patients is known and their medical needs adequately coordinated, and standardized medical records in order to ease the transfer of information between departments and aid health professionals.

Novati et al. ${ }^{5}$ significantly reduced outliers (from 6.3 to $5.4 \%$ ) by implementing an algorithm, supporting rational outward allocation of patients and difficult discharges.

\section{DISCUSSION}

To our knowledge, this is the first systematic review on outliers on medicine wards. The literature suggests a possible trend towards increased mortality and hospital readmissions among 


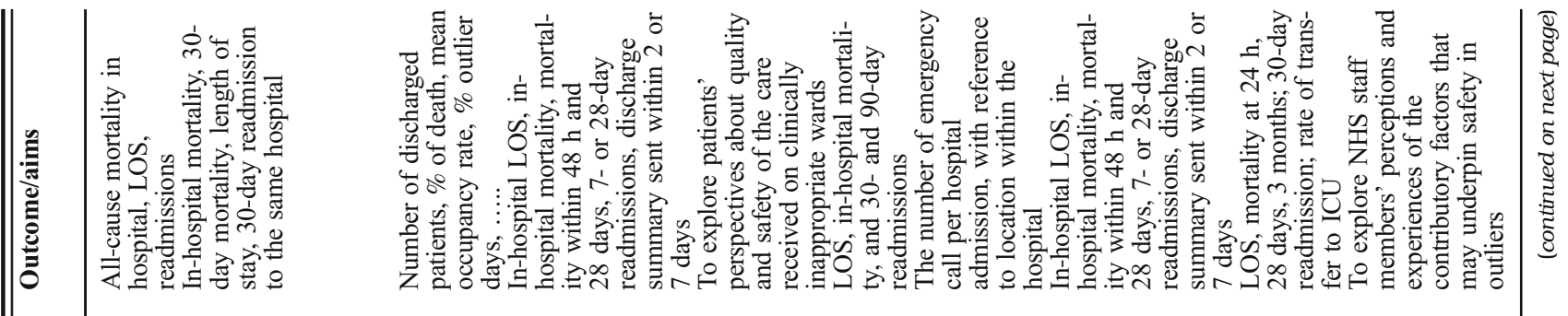

눙 윰

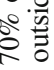

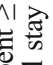

क्षेत्क

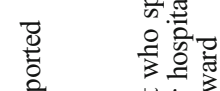

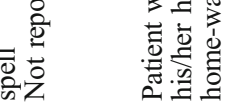

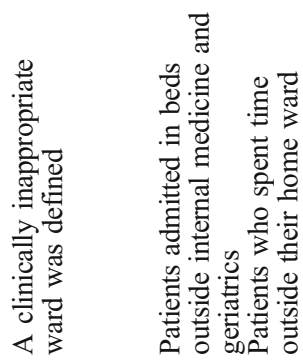

岁胨

$\Xi 4$

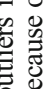

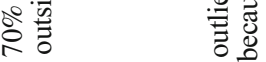

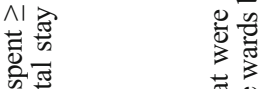

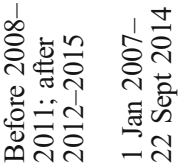

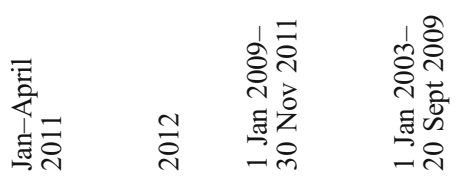

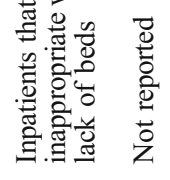

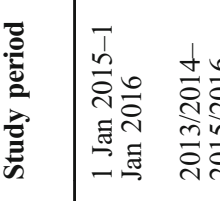

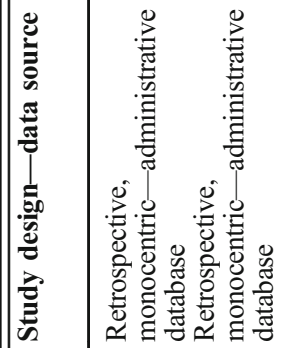

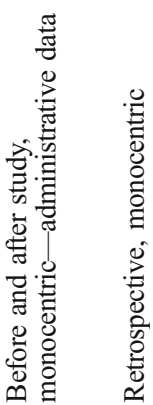

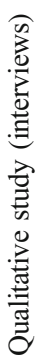

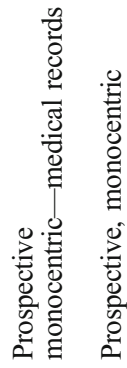

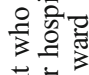

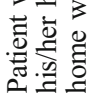

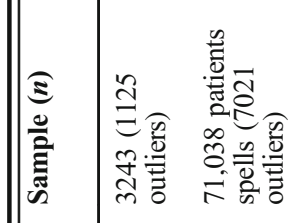

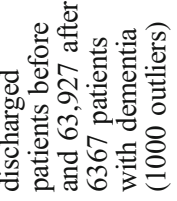

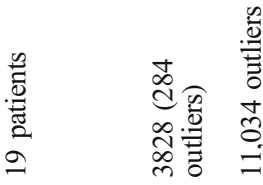

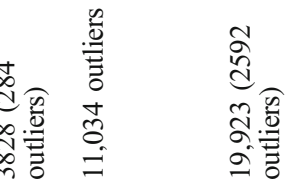

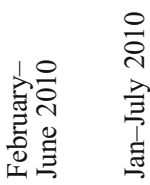

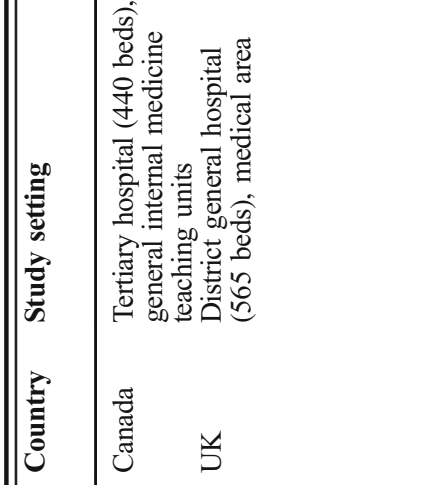

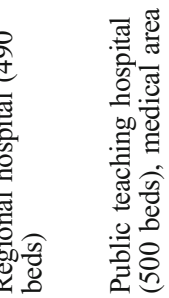

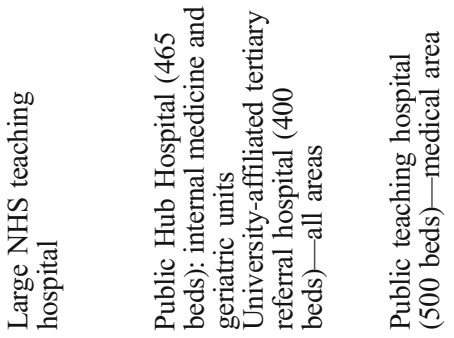

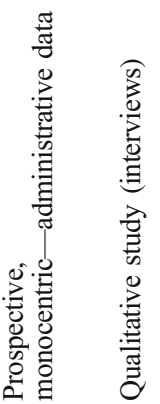

뭉

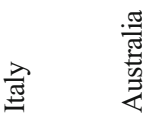

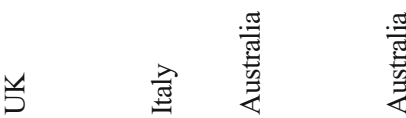

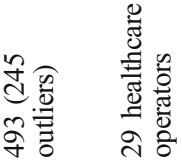

赔

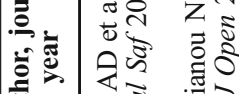

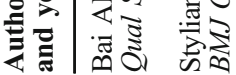

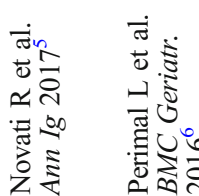

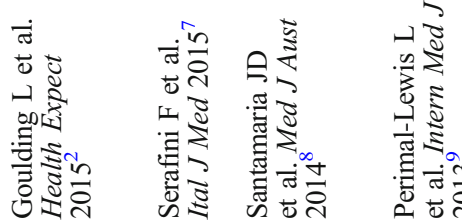

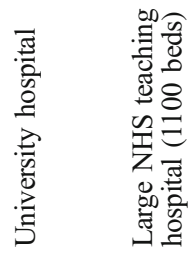

苋苞

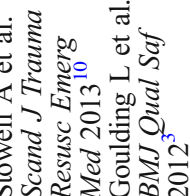




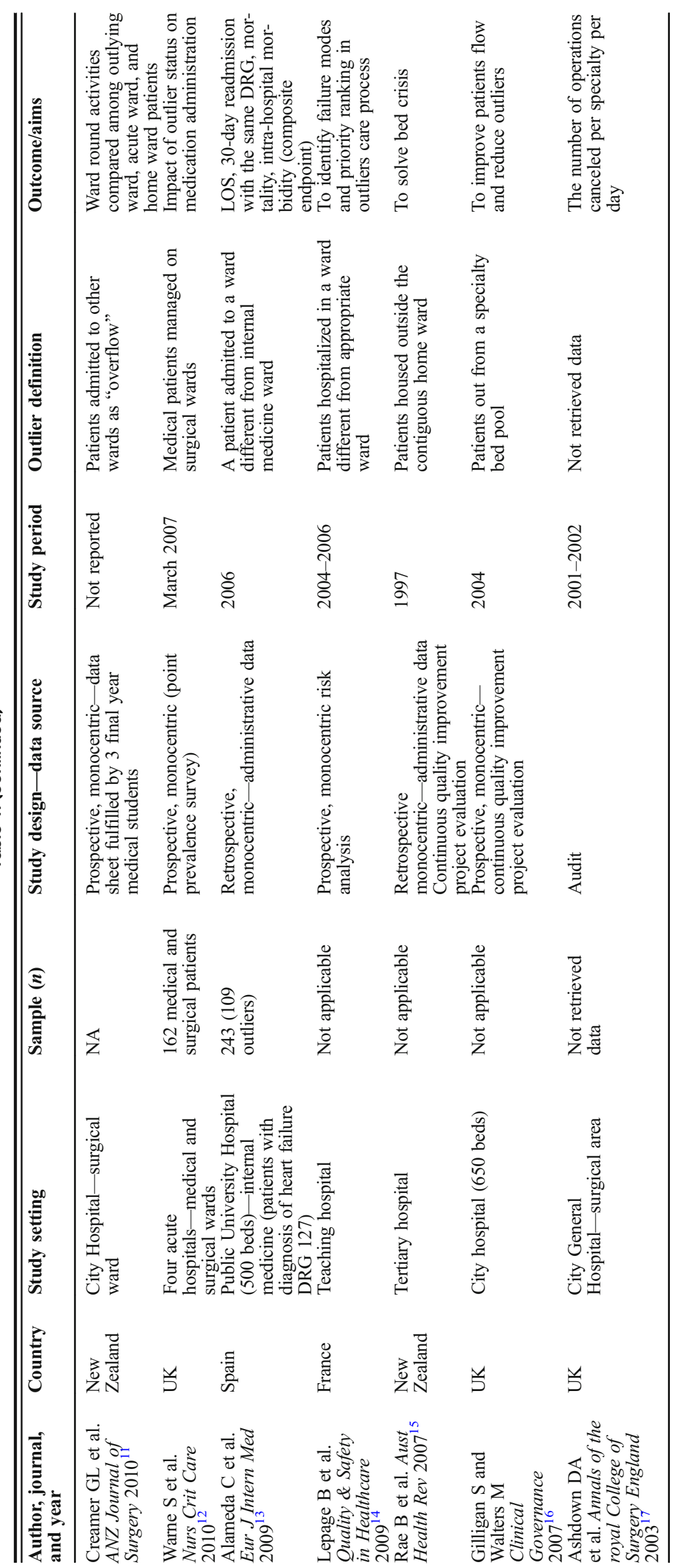


Table 2 Results of eligible studies grouped in six thematic categories

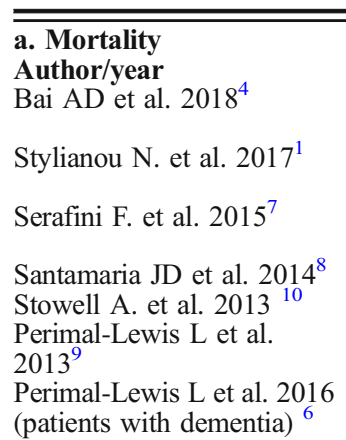

Alameda C. et al. $2009^{13}$

b. Length of stay (LOS)

Author/year

Bai AD et al. $2018^{4}$

Stylianou N et al. $2017^{1}$

Serafini F. et al. $2015^{7}$

Perimal-Lewis et al. $2013^{9}$

Stowell A. et al. $2013^{10}$

Alameda C. et al. $2009^{13}$

c. Readmissions

Author/year

Stylianou et al. $2017^{1}$

Serafini F et al. $2015^{7}$

Perimal-Lewis et al. $2013^{9}$

Stowell A. et al. $2013^{10}$

Alameda et al. 2009

d. Other indicators

Author/year

Serafini F et al. $2015^{7}$

Stowell A. et al. $2013^{10}$

Perimal-Lewis et al. $2013^{9}$

Creamer et al. $2010^{11}$

Alameda et al. $2009^{13}$ Ashdown et al. $2003^{17}$

Santamaria JD et al. $2014^{8}$ Warne S et al. 2010

e. Perceived quality and sa Author/year

Goulding L. et al. 2012-

$2015(2,3)$

\section{f. Safety issues}

Author/year

Rae B. et al. $2007^{16}$

Lepage B et al. $2009^{5}$
Measure

Hazard ratio (HR)

Odds ratio (OR)

Hazard ratio (HR)

58.158 in-hospital mortality rate

Mortality rate at $24 \mathrm{~h}$

In-hospital mortality rate; in-hospital mortality within $48 \mathrm{~h}$

In-hospital mortality rate; mortality rate within $48 \mathrm{~h}$; odds ratio

In-hospital mortality

Sample $(n)$ and measure

LOS in days

LOS in days

LOS in days

LOS in hours

LOS in days

LOS in days

Measure

Odds ratio

Rate at 90 days

Rate at 7 and 28 days

Rate at 28 days

Rate at 30 days

Indicator

Type of patients less allocated off-ward

VTE prophylaxis

Number of blood and imaging tests (SD)

ER length of stay

Discharge summary completion within 2 days

Discharge summary completion within 7 days

Mean consultation time

Mean discussion time

Time spent to traveling between wards

In-hospital morbidity*

Rate of canceled surgeries

$\%$ calls to in-hospital emergency team

Rate of not administered medications in surgical wards

\section{Indicator}

NA (qualitative study)
Results (outliers vs non outliers)

$\uparrow 3.42$ on admission decreases by

0.97 per day

$=$ outliers are not associated with

in-hospital mortality (OR 0.983)

$\uparrow$ for outliers in surgical wards (1.8, $\quad p<0.05$

$1.2-2.595 \% \mathrm{CI})$

$\uparrow(2.57 \%$ vs $1.12 \%)$

$\downarrow(0.00 \%$ vs $0.84 \%)$

$4.5 \%$ vs $3.5 \%$

$50.4 \%$ vs $22.4 \%$

$\uparrow(9.6 \%$ vs $7.9 \%)$

$\uparrow(3.2 \%$ vs $1.16 \%)$

$\uparrow$ OR 1.973 ; 95\% CI 1.158-3.359)

$\downarrow(17 \%$ vs $22 \%)$

$p<0.0001$

$p=0.773$

$p<0.001$

$p<0.05$

$p=0.014$

$p<0.001$

$p=0.072$

$p=0.000$

$p=0.012$

$p=0.412$

Results (outliers vs non outliers)

$=(5.31$ vs 5.97 days $)$

$\uparrow$ (7 vs 3 days)

$=(9.8 \mathrm{vs} 10$ in internal medicine

wards; 13 for both in geriatric

wards)

$\downarrow(110.7 \mathrm{~h}$ vs $141.9 \mathrm{~h})$

$\uparrow$ (8 vs 7 days)

$\uparrow(11.8$ vs 9.2 days $)$

p

$p=0.1119$

$p<0.001$

$p$ not

reported

$p<0.001$

$p=0.04$

$p=0.001$

Results (outliers vs non outliers)

$\uparrow$ (odds at 30 days at univariate

analysis not confirmed by

multivariate)

$\uparrow(26.1$ vs $14.2 \%)$

1.2 vs $2 \%$ at 7 days

2.1 vs $4.9 \%$ at 28 days

$\uparrow(27$ vs $17 \%)$

$\uparrow(15$ vs $10 \%)$

Results (outliers vs non outliers)

Respiratory patients

42 vs $52 \%$

5.13 vs 4.59

1.65 vs 1.41

6.3 vs $5.3 \mathrm{~h}$

40.7 vs $61.2 \%$

$64.3 \%$ vs $78 \%$

$152^{\prime \prime}$ vs $136^{\prime \prime}$

$25^{\prime \prime}$ vs $14^{\prime \prime}$

$18 \%$

$\boldsymbol{p}$
$p=0.09$

$24 \%$ vs $18 \%$

$14.8 \%$

$\uparrow$ by $53 \%$

$\uparrow(100 \%$ vs $74 \%)$

Results (outliers vs non outliers)

Patients and health operators reported many safety threats in outliers $p<0.0001$

$p=0.003$

$p<0.001$

$p=0.008$

$p=0.234$

Not

applicable

$p=0.03$

Not

$p<0.001$

$p<0.001$

$p<0.001$

Not

reported

$p=0.254$

Not

$p<0.001$

$p<0.001$

p

applicable reported

applicable

\section{Safety issues}

Staff factors: too many consultants - large variation in clinical practice

Process factors within the control of the service: adverse events; ward rounds miss patients; patients not seen at weekends; lack of communication across disciplines; lack of a diagnosis; all diagnoses not dealt with from the start of the admission; too many patients under a single team; interrupted ward rounds by being paged for non-urgent requests. Emergency department care

Nurse responsible for finding beds for outlying patients not available

Inaccurate or out-of-date information about bed occupancy in the hospital

Best compromise between outlier's pathology and outlying ward's specialty not taken into account at disposal decision time

Outlying ward contact, called by emergency department before transfer agreement, varying from ward to ward (duty doctor, charge nurse, nurse)

Person in charge of admission agreement in outlying ward not contactable

Wrong information given to outlying wards about outlying patients 
Emergency department contact for outlying patients not known by outlying wards or appropriate specialty wards Appropriate specialty staff not informed of hospitalization of outliers who should be in their charge

Transfer from emergency department to outlying ward

Final diagnosis or final clinical assessment not made in emergency department, potentially resulting in transfer of patients in unstable condition; emergency department porters not available for patient transfer; bad communication between emergency department and outlying wards about time of transfer; bad communication between emergency department and porters regarding name of outlying ward; patient transferred to outlying ward without medical record First day of hospital care

Final diagnosis or final clinical assessment not entered into emergency department medical record

Medical or nursing records varying from department to department

No medical record used for outlying patients

Bed not yet available at time of admission to outlying ward

Delayed admission of patients scheduled for non-urgent problems or elective procedures

Doctors in outlying wards not aware of new outliers hospitalized in their wards

No defined contact in outlying wards (nurse, charge nurse, or doctor) to call a specialist doctor in appropriate specialty ward

No traceability of calls from outlying wards to specialist doctors; in appropriate specialty wards, no identification of specialist doctors responsible for care of outlying patients falling within their sphere of competence

Specialist doctor in appropriate ward not easily contactable; lack of information or prescription from a specialist doctor in appropriate ward to nurses and doctors in outlying ward; no specialist medical and nursing care; diagnostic tests not ordered by a doctor from appropriate specialty; no specialist interpretation of diagnostic tests performed on outlying patients; no specialist information given to outlying patients and their families; no systematic meeting or information transmission between doctors in outlying wards and doctors in appropriate specialty wards; inappropriate nursing care provided to outlying patients

Care in outlying ward from the second day of hospitalization until the day before discharge

No specialist follow-up; results of diagnostic tests not systematically transmitted to a specialist doctor in appropriate ward; no specialist information given to outlying patients and their families

Day of discharge

Information about discharge and follow-up of outlying patients not given by a specialist doctor from the appropriate ward

Information in medical record and discharge documents not completed by a specialist doctor from the appropriate ward Transport forms and prescriptions not completed by a specialist doctor from the appropriate ward Follow-up of outlying patients not scheduled by specialist doctors from appropriate wards

\section{g. Solutions}

Author/year

Novati R. et al. $2017^{5}$

Lepage B et al. $2009^{14}$

Gilligan S et al. $2007^{16}$

Rae B. et al. $2007^{15}$

\section{Solutions}

Algorithm supporting rational outward allocation of patients and difficult discharges

Identification of medical doctor and nurse coordinator for outliers, use of standardized medical records

"Physician of the week", discharge facilitator, "quick and sick" ward

Discharge planning, increase of transfers from general internal medicine to geriatrics, implementation of a consultant-led ward round 7 days a week

\section{Results}

Outlier days fell from 6.3 to $5.4 \%$

Not reported

Reduction of HospitalStandardized Mortality Rate (HSMR)

Outlier bed crises solved $p$
$p=0.000$

Not applicable

Not reported

Not applicable

*Intra-hospital infection (urinary, respiratory, bacteremia, or others beginning $48 \mathrm{~h}$ after admission), intra-hospital hemorrhage (digestive, urinary, or others), and intra-hospital venous thromboembolism

outliers, though the data was too heterogeneous to pool. The majority of the studies ${ }^{4,6-9}$ found a significant increase of inhospital mortality rate or risk, especially in the first 2 days when patients are medically more active. Data about 30 - or $90-$ day mortality are sparse. Readmissions were evaluated at different intervals (from 7 to 90 days after discharge) in the collected studies. Three out of five documented a larger proportion of 28-day readmissions among outliers; the fourth study documented an increased risk of readmission, but only at univariate analysis. Data about length of stay (LOS) were too inconsistent across the studies to reach any meaningful conclusions.

In addition to being too heterogeneous for pooling, most of the study designs among the included papers were poor, mainly monocentric, retrospective, based on administrative data, and underpowered. ${ }^{1,13}$ On the other hand, the inconsistency of results can be due also to different contexts. For example, the habit of moving stable patients outside to admit unstable ones or planning early the discharge, different availability of community facilities, health services, and social support can contribute to discordance. Nevertheless, delay between admission and medical evaluation, discontinuity of care, errors or delay in tests request/execution, inadequate communication between ward teams, less familiarity with monitoring and treatment by hosting team, and nosocomial complications can variously affect mortality, length of stay, and readmission rate. Worrisome is the literature that suggests specialized wards lead to better outcomes from some conditions, such as stroke, renal failure, burns, asthma, gastrointestinal bleeding, trauma, and cancer. ${ }^{18-23}$

Evidence about other indicators such as proportion of elective surgeries canceled, ${ }^{17}$ thrombo-prophylaxis, in-hospital infections or in-hospital bleedings, number and appropriateness of investigations, calls to intra-hospital emergency team, and missed medications is limited, but there are other possible drawbacks to being boarded. Moving patients has been shown 
to increase the risk of healthcare-associated infection (HCAI). ${ }^{24}$

Two studies exploring patient and provider satisfaction both suggest a perception of reduced quality and safety. ${ }^{2,3}$ This can be due to travel time, to lack of established relationships between providers and nurses on the outlying wards, and to worry about patients that are not immediately accessible. The hosting nursing team also feels a sense of inadequacy due to less expertise in the management of outlier's health problems. Patients feel they do not belong to any ward, feel forgotten, are worried about errors due to staff inexperience, miscommunication, or resource unavailability, and dislike transfers between wards.

All suggested solutions ${ }^{5,14-16}$ are multi-component as the problem is complex and needs a system approach and have not been rigorously studied, yet. The "best" solutions are likely to be tailored to the specifics of the individual systems.

\section{CONCLUSIONS}

Though literature evidence is quite limited and heterogeneous, the outlier status may be associated with worse outcomes. Certainly, patients and health professionals are dissatisfied. The reported solutions are targeted to locally identified problems and have not been rigorously studied.

There is a need to reach a universally accepted definition of outlier, to adequately measure the effect of outlier status on clinical and safety outcomes, and to develop validated tools to analyze and manage a phenomenon that could negatively impact on care and organizational outcomes.

To this aim, FADOI (the Federation of the Associations of Hospital Internists) has planned a multicenter, prospective, wellsized study comparing mortality rate and adverse event rate in outliers and non-outliers, named "Safety Issues and SurvIval For medical Outliers" (SISIFO) study (NCT03651414) that will start at the end of 2018 .

Corresponding Author: Micaela Regina, MD; SS Risk Management ASL5 Liguria, La Spezia, Italy (e-mail: micaela.laregina@asl5.liguria.it).

\section{Compliance with Ethical Standards:}

Conflict of Interest: The authors declare that they do not have a conflict of interest.

\section{REFERENCES}

1. Stylianou N, Fackrell R, Vasilakis C. Are medical outliers associated with worse patient outcomes? A retrospective study within a regional NHS hospital using routine data. BMJ Open 2017;7:e015676.

2. Goulding $\mathbf{L}$, Adamson $\mathbf{J}$, Watt $\mathbf{I}$, et al. Lost in hospital: a qualitative interview study that explores the perceptions of NHS inpatients who spent time on clinically inappropriate hospital wards. Health Expect 2015;18:982-94.
3. Goulding $\mathbf{L}$, Adamson $\mathbf{J}$, Watt $\mathbf{I}$, et al. Patient safety in patients who occupy beds on clinically inappropriate wards: a qualitative interview study with NHS staff. BMJ Qual Saf 2012;21:218-24.

4. Bai AD, Srivastava S, Tomlinson GA, Smith CA, Bell CM, Gill SS. Mortality of hospitalized Internal medicine patients bedspaced to noninternal medicine inpatient units: retrospective cohort study, BMJ Qual Saf. 2018;27:11-20.

5. Novati R, Papalia R, Peano L, Gorraz A, Artuso L, Canta MG, Del Vescovo G, Galotto C. Effectiveness of an hospital bed management model: results of four years of follow-up. Ann Ig. 2017;29(3):189-196.

6. Perimal-Lewis L, Bradley $\mathbf{C}$, Hakendorf $\mathbf{P H}$, Whitehead $\mathbf{C}$, Heuzenroeder L, Crotty $\mathbf{M}$. The relationship between in-hospital location and outcomes of care in patients diagnosed with dementia and/or delirium diagnoses: analysis of patient journey. BMC Geriatr. 2016;16(1): 190 .

7. Serafini F, Fantin G, Brugiolo R, Lamanna O, Aprile A, Presotto F. Outlier admissions of medical patients: prognostic implications of outlying patients. The experience of the hospital of Mestre. Ital J Med. 2015;9:299-302.

8. Santamaria JD, Tobin AE, Anstey MH, Smith RJ, Reid DA. Do outlier inpatients experience more emergency calls in hospital? An observational cohort study. Med J Aust. 2014;200:45-8.

9. Perimal-Lewis L, Li JY, Hakendorf PH, Ben-Tovim DI, Gin S, Thompson CH. Relationship between in-hospital location and outcomes of care in patients of a large general medical service. Intern Med J. 2013;43(6):712-6

10. Stowell A, Claret PG, Sebbane M, Bobba X, Boyard C, Grandpierre RG, Moreau A, de le Coussaye J-E. Hospital out-lying through lack of beds and its impact on care and patient outcome. Scand J Trauma Resusc Emerg Med 2013;21:17.

11. Creamer GL, Dahl A, Perumal D, Tan G, Koea JB. Anatomy of the ward round: the time spent in different activities. ANZ J Surg. 2010; 80 (12): 930-932.

12. Warne S, Endacott R, Ryan H, Chamberlain W, Hendry J, Boulanger C, Donlin N. Non-therapeutic omission of medications in acutely ill patients. Nurs Crit Care. 2010;15(3):112-7.

13. Alameda C, Suárez C. Clinical outcomes in medical outliers admitted to hospital with heart failure. Eur J Intern Med. 2009;20:764-7.

14. Lepage B, Robert R, Lebeau M, Aubeneau C, Silvain C, Migeot V. Use of a risk analysis method to improve care management for outlying inpatients in a University hospital. Qual Saf Health Care. 2009; 8:441445

15. Rae B, Busby W, Millard PH. Fast-tracking acute hospital care-from bed crisis to bed crisis. Aust Health Rev. 2007;31(1):50-62.

16. Gilligan S, Walters M. Quality improvements in hospital flow may lead to a reduction in mortality. Clin Gov Int J. 2007; 13(1):.26-34.

17. Ashdown DA, Williams D, Davenport K, Kirby RM. The impact of medical outliers on elective surgical lists. Ann R Coll Surg Engl. 2003;85: $46-47$.

18. Sanderson JD, Taylor RF, Pugh S, Vicary FR. Specialized gastrointestinal units for the management of upper gastrointestinal hemorrhage. Postgrad Med J. 1990;66:654-56

19. Bucknall CE, Robertson C, Moran F, Stevenson RD. Management of asthma in hospital: a prospective audit. Br Med J. 1988;296:1637-9.

20. Mayor S. Stroke patients prefer care in specialist units. Br Med J. 2005;331:130

21. Wright N, Shapiro LM, Nicholson J. Treatment of renal failure in a nonspecialist unit. Br Med J, 1980; 281: 117-118.

22. Lloyd JM, Elsayed S, Majeed A, Kadambande S, Lewis D, Mothukuri $\mathbf{R}$, Kulkarni R. The practice of out-lying patients is dangerous: a multicentre comparison study of nursing care provided for trauma patients. Injury. 2005;36(6):710-3.

23. Mohan S, Wilkes LM, Ogunsiji O. Walker A. Caring for patients with cancer in non-specialist wards: the nurse experience. Eur J Cancer Care. 2005; 14:256-263.

24. Clements A, Halton $K$, Graves N, Pettitt A, Morton A, Looke D, Whitby M. Overcrowding and understaff- ing in modern health-care systems: key determinants in meticillin- resistant Staphylococcus aureus transmission. Lancet Infect Dis. 2008;8: 427-434.

Publisher's Note Springer Nature remains neutral with regard to jurisdictional claims in published maps and institutional affiliations. 